\title{
Características clínicas e oftalmológicas de indivíduos com necessidades especiais institucionalizados no estado de Pernambuco, Brasil
}

\section{Clinical and ophthalmological findings in subjects with special needs institutionalized in Pernambuco, Brazil}

Priscila de Almeida Jorge ${ }^{1}$, Liana Maria de Oliveira Ventura 2 ${ }^{2}$ Cínthia Rubia Teló ${ }^{3}$, Andrea Gondim Leitão Sarmento ${ }^{4}$, Patrícia Rios da Silva Rego ${ }^{5}$

\section{Resumo}

Objetivo: Descrever as características clínicas e oftalmológicas encontrados em indivíduos institucionalizados, com necessidades especiais no estado de Pernambuco. Métodos: Realizou-se projeto multidisciplinar em 37 pacientes, com necessidades especiais, institucionalizados na Comunidade Rodolfo Aureliano (CRAUR), unidade da Fundação da Criança e do Adolescente (FUNDAC) em Recife-PE. A faixa etária dos indivíduos institucionalizados variou de seis a 30 anos, média de 18,8 $\pm 5,1$ anos. O sexo masculino foi mais prevalente com 35 indivíduos (94,5\%). Consideraram-se como indivíduos institucionalizados, aqueles que vivem abrigados em instituições mantidas pelo Estado. Foi feito estudo de corte transversal, verificando-se os principais achados clínicos e oftalmológicos destes indivíduos. Realizou-se o exame oftalmológico completo nos casos, com boa colaboração e de acordo com protocolo pré-estabelecido. Resultados: Uso de álcool na gravidez foi relatado por nove mães $(24,3 \%)$. Verificou-se a presença de retardo mental grave em 22 casos $(59,4 \%)$, paralisia cerebral e epilepsia em sete $(18,9 \%)$ indivíduos cada. Foi possível a realização do exame oftalmológico completo em 24 indivíduos (64,8\%). Detectou-se cegueira legal em dez casos $(41,6 \%)$. Os diagnósticos oftalmológicos mais encontrados foram ambliopia, erro refracional e phthisis bulbi em três casos (12,5\%) cada. Estrabismo foi encontrado em dois casos $(8,3 \%)$. Conclusão: Os achados oftalmológicos revelaram a importância do exame oftalmológico em indivíduos com necessidades especiais institucionalizados. A associação com múltiplas deficiências, tais como: doenças neuropsiquiátricas, contribuiu para o maior grau de dificuldade na avaliação e condução dos casos.

Descritores: Síndrome alcoólica fetal; Anormalidades múltiplas; Pessoas com deficiências; Saúde da criança institucionalizada; População institucionalizada; Anormalidades do olho

\footnotetext{
1 Médica, aluna do curso de especialização em Oftalmologia da Fundação Altino Ventura - FAV - Recife (PE), Brasil;

2 Doutora, Coordenadora do curso de especialização em Oftalmologia da Fundação Altino Ventura. Coordenadora do Departamento de Oftalmologia Pediátrica e Estrabismo do Hospital de Olhos de Pernambuco (HOPE) - Recife (PE), Brasil;

${ }^{3}$ Médica, Aluna do Curso de Fellow em Oftalmologia Pediátrica, Estrabismo e Visão Subnormal da Fundação Altino Ventura - FAV -

Recife (PE), Brasil.

${ }^{4}$ Médica, aluna do Curso de especialização em Oftalmologia da Fundação Altino Ventura - FAV - Recife (PE), Brasil;

${ }^{5}$ Oftalmologista especializada em Oftalmologia Pediátrica, Estrabismo e Visão Subnormal da Fundação Altino Ventura - FAV - Recife (PE), Brasil.

Trabalho realizado na Comunidade Rodolfo Aureliano (CRAUR), unidade da Fundação da Criança e do Adolescente (FUNDAC) Recife (PE), Brasil.

Não existe conflito de interesse na realização da investigação.

Recebido para publicação em: 13/4/2010 - Aceito para publicação em 1/2/2011
} 


\section{INTRODUÇÃO}

A família representa o espaço prioritário e o alicerce sobre os quais ocorrem as oportunidades de socialização, bem-estar e proteção da criança e onde são estabelecidas as relações humanas ${ }^{(1)}$. Se uma criança cresce em situação irregular (afastada da vida familiar), pressupõe-se que sua base de segurança tende a desaparecer, o que pode prejudicar suas relações com os outros, havendo, assim, prejuízos nas demais funções do seu desenvolvimento. Crianças que nascem com necessidades especiais exigem um cuidado especializado, o que nem sempre é oferecido pelos seus cuidadores. Frequentemente ocorre negligência parental e abandono, gerando o eventual acolhimento em instituições ${ }^{(2)}$.

A visão desenvolve-se principalmente nos primeiros seis anos de vida. A sua plasticidade sensorial é maior nos dois primeiros anos. Isto é até esta idade qualquer obstáculo ao desenvolvimento da visão causa diminuição rápida da acuidade visual, assim como o tratamento neste período promove pronta recuperação. A visão depende do desenvolvimento da retina, das vias ópticas e do córtex visual, aparecendo diminuída em toda afecção dessas estruturas ou quando o próprio desenvolvimento das competências neuronais se faz imperfeitamente ${ }^{(3)}$.

A visão é o sentido mais importante para o desenvolvimento do ser humano, uma vez que proporciona a interação com o ambiente. Sua diminuição ou ausência pode reduzir a aquisição ou manutenção das habilidades motoras, dificultando os movimentos ativos e seguros. As pessoas que apresentam necessidades especiais requerem maior estimulação para seu desenvolvimento, exercendo a visão um papel de destaque para aquisição de informações e estimulação adequada ${ }^{(4)}$.

Indivíduos institucionalizados com necessidades especiais apresentam anormalidades oftalmológicas em maior frequência quando comparados com pessoas normais. Destacam-se maior número de anormalidades de fundo de olho, erros refrativos, de pálpebras e da motilidade ocular ${ }^{(5)}$. No Brasil e no mundo, estudos têm demonstrado maior frequência de ambliopia, erros refrativos e estrabismo quando comparados com indivíduos normais ${ }^{(4-7)}$. Sendo assim, as alterações oculares de pessoas com necessidades especiais devem ser reconhecidas e tratadas de maneira adequada, por profissionais capacitados, utilizando recursos especiais. O diagnóstico precoce das anormalidades e o tratamento adequado podem significar importante melhora nos padrões globais de desenvolvimento e maior resposta às terapias à que são submetidos, consequentemente melhor qualidade de vida ${ }^{(6)}$.
No Brasil, existe importante carência de dados oftalmológicos quanto a pacientes com necessidades especiais institucionalizados. Porém, sabe-se que quando comparados com indivíduos sem as mesmas necessidades, os achados oftalmológicos mostram maior relevância.

O presente estudo teve como objetivo investigar os principais achados oftalmológicos encontrados em projeto realizado em indivíduos institucionalizados, com necessidades especiais, verificando a associação com outros tipos de deficiências.

\section{Métodos}

O estudo fez parte de um projeto amplo realizado em indivíduos institucionalizados do estado de Pernambuco, pela equipe multidisciplinar do Centro de Reabilitação Visual e/ou Múltiplas Deficiências da Fundação Altino Ventura (FAV) Pernambuco, e pelo Departamento de Oftalmologia da Universidade de Sahlgrenska, Universidade de Gotemburgo, Suécia.

Descreveu-se a experiência obtida com o projeto realizado em 37 indivíduos com necessidades especiais, institucionalizados na Comunidade Rodolfo Aureliano (CRAUR) da Fundação da Criança e do Adolescente (FUNDAC) em Recife-PE. Consideraram-se como indivíduos institucionalizados, aqueles que viviam abrigados em instituições mantidas pelo Estado. A faixa etária dos indivíduos variou de seis a 30 anos, média de 18,8 \pm 5,1 anos. Trinta e cinco indivíduos eram do sexo masculino e dois do feminino.

Trata-se de estudo de corte transversal, clínico, descritivo com amostra de conveniência.

A CRAUR é uma das dez unidades protetoras mantidas pela FUNDAC no Estado, que tem por finalidade abrigar crianças e adolescentes abandonados por familiares ou que a Justiça retirou o poder da família, portadores de deficiências mentais isoladas ou associadas a outras deficiências. De acordo com o estatuto interno da Instituição, o atendimento é pré-estabelecido para meninos e meninas até 18 anos, mas por carência de abrigos ou outras instituições afins, acaba por abrigar adultos também. A CRAUR abriga indivíduos do sexo feminino apenas em casos de deficiência mental grave, a fim de evitar ou minimizar problemas da ordem sexual entre os mesmos.

A colaboração da diretoria e toda equipe da CRAUR foi de fundamental importância para a realização deste estudo, tendo em vista as múltiplas deficiências apresentadas pelos pacientes e as dificuldades inerentes à avaliação dos mesmos.

Todas as etapas do estudo foram realizadas nas dependências da CRAUR, no espaço que já é familiari- 
zado pelos indivíduos, contando com a ajuda da equipe multidisciplinar da própria instituição, com a qual eles já estavam habituados. Coube a FAV fornecer todos os equipamentos oftalmológicos e uma equipe multidisciplinar composta de: oftalmologistas, pediatra, psicóloga, assistente social, assistentes oftálmicos e voluntários.

Durante as etapas do exame uma equipe de voluntários da FAV realizava atividades recreativas diversas e doação de lanches.

No início da avaliação foi realizada uma anamnese dirigida aos cuidadores, verificando-se os dados existentes com relação à história pregressa e psicossociais. Verificou-se a etiologia das múltiplas deficiências dos indivíduos nos prontuários médicos da própria instituição.

Todos os indivíduos internados na instituição foram examinados pela equipe da FAV, obedecendo a um protocolo previamente estabelecido, constando de dados de anamnese, incluindo antecedentes pessoais e familiares, exame clínico e oftalmológico.

Dentro do possível, e de acordo com a colaboração de cada um, realizou-se o exame oftalmológico completo nos indivíduos. Entretanto, alguns casos efetivouse apenas a ectoscopia devido à pouca colaboração.

Para a aferição da acuidade visual utilizou-se o Teste de Teller (Teller Acuity Cards - para crianças de até três anos ou nos casos de pouca colaboração). Os indivíduos com boa colaboração foram examinados através da tabela de Snellen de figuras ou de letras.

O exame da motilidade ocular foi feito utilizando-se os testes de Hirschberg e Krimsky. A retinoscopia foi feita sob cicloplegia. Para o exame de fundoscopia utilizou-se o oftalmoscópio binocular indireto.

Classificou-se a acuidade visual conforme o Código Internacional de Doenças - CID-10 da OMS, sendo normal a acuidade visual de 20/20, próximo do normal $\leq$ a 20/60, baixa visão $\leq 20 / 70$ até $20 / 400$, e cegueira $\leq$ a 20/400 até sem percepção luminosa (SPL) ${ }^{(8)}$.

Consideraram-se como erros refrativos passíveis de correção a hipermetropia $\leq 2,00 \mathrm{D}$, miopia $\leq 1,00 \mathrm{D}$ e/ ou astigmatismo $\leq 0,75 \mathrm{D}$.

Os dados categóricos foram resumidos através de suas frequências absolutas e relativas, apresentados através de tabelas e gráficos.

O projeto de investigação foi aprovado pelo Comitê de Ética em Pesquisa da Fundação Altino Ventura e pela comissão coordenadora da FUNDAC.

\section{Resultados}

História de uso de álcool pela mãe na gravidez ocorreu em nove indivíduos $(24,3 \%)$, dentre estes se verificou retardo mental em cinco $(55,5 \%)$, cegueira em quatro (44,4\%), sendo que três destes também apresentavam retardo mental.

Foi feito uso de cocaína por uma genitora $(2,7 \%)$, seu filho era portador da síndrome de Sturge Weber e microcefalia.

Uma das mães (2,4\%) tinha HIV positivo, e seu filho apresentava retardo mental grave e paralisia cerebral.

Dos 37 indivíduos institucionalizados, 22 (59,4\%) apresentavam retardo mental grave. Epilepsia e paralisia cerebral foram observadas em sete casos $(18,9 \%)$. Dois indivíduos apresentaram estrabismo $(5,4 \%)$, e havia um portador de miopia em associação com coroidose miópica $(2,7 \%)$.

Uma variedade de outras deficiências foi encontrada ora isolada ora associada, tais como: dismorfismo facial em seis indivíduos $(16,2 \%)$, autismo em três $(8,1 \%)$. Deficiência auditiva, hidrocefalia, microcefalia e esquizofrenia foram encontradas em dois indivíduos $(2,7 \%)$ cada. Os seguintes achados foram detectados cada um, isolados ou associados, em um indivíduo: miopatia congênita, desnutrição, luxação congênita do quadril, encefalopatia crônica evolutiva, encefalopatia póstraumatismo cranioencefálico(TCE), hepatite B, esclerose tuberosa, distrofia muscular progressiva, transtorno psicótico, lúpus eritematoso sistêmico e meningite meningocócica. Também foram encontradas síndrome de Down em dois casos $(5,4 \%)$, e síndromes de SturgeWeber, de Lennox-Gastaut e síndrome da rubéola congênita em um caso cada $(2,7 \%)$. O portador da síndrome de Lennox-Gastaut havia tido um episódio de meningite de etiologia não especificada. Ambos portadores da síndrome de Down apresentavam retardo mental grave, sendo que um deles também apresentava hepatite B. O portador da síndrome da rubéola congênita apresentava déficit visual e auditivo bilateral, retardo mental grave e dismorfismo facial, além dos achados oftalmológicos de microftalmia e phthisis bulbi (atrofia do bulbo do olho) (Tabela 1).

O exame oftalmológico completo pôde ser realizado em 24 (64,9\%) indivíduos. Em 13 (35,1\%) realizou-se apenas a ectoscopia devido à pouca colaboração; destes, dez $(77 \%)$ apresentavam retardo mental grave.

$\mathrm{Na}$ avaliação dos achados fenotípicos dos 37 indivíduos, verificaram-se oito casos $(21,6 \%)$ de base nasal alargada, e quatro $(10,8 \%)$ com filtro anormal. Dentre os oito indivíduos com a base nasal alargada, três tinham história materna de uso de álcool durante a gestação, e destes, dois também apresentavam filtro anormal. Ptose e achatamento da face média foram encontrados em um caso cada $(2,7 \%)$. Houve um caso de hipoplasia do nervo óptico acompanhado de base nasal alargada, epicanto e cegueira legal, sem relato de uso de álcool durante a gestação. 
Dentre os 24 indivíduos que permitiram o exame da acuidade visual, havia dez casos $(41,6 \%)$ com cegueira legal em ambos os olhos, incluindo dois casos (8,3\%) com phthisis bulbi bilateral (Tabela 2).

Os achados oftalmológicos mais frequentes foram a ambliopia, Phthisis bulbi e os erros refrativos passíveis de correção foram vistos em três casos $(12,5 \%)$ respectivamente, sendo o mais encontrado a miopia, vista em dois pacientes $(8,3 \%)$ (Tabela 3$)$.

\section{DiscussÃo}

A amostra deste estudo é composta de indivíduos institucionalizados com necessidades especiais, abrigados sob a custódia do estado de Pernambuco. Na maioria dos casos há relatos de maus tratos ou negligências dos genitores, resultando em uma deficiente história pregressa.

Dentre os achados clínicos encontrados o retardo mental grave apresentou o maior índice, seguido pela epilepsia e paralisia cerebral. Além destes, mas em menor proporção, encontrou-se retardo mental leve a moderado, dismorfismo facial, autismo, microcefalia. Essas alterações são semelhantes às encontradas em outros estudos de indivíduos institucionalizados ${ }^{(5,9-13)}$.

O uso de álcool na gravidez, relatado por nove genitoras, é uma realidade condizente com a conhecida no país, no qual este índice vem aumentando significativamente nos últimos anos. No Brasil, estima-se que 6/ 1000 mulheres façam uso de álcool durante a gestação ${ }^{(9)}$. As alterações encontradas neste grupo de indivíduos são comuns às manifestações oftalmológicas da síndrome alcoólica fetal (SAF), tais como: epicanto, filtro nasal hipoplásico, base nasal alargada, achatamento da face média, baixa acuidade visual, hipoplasia do nervo óptico, miopia e ptose, em associação com uso de álcool durante a gestação ${ }^{(9,14-22)}$. Porém, não foi confirmado nenhum caso de SAF dentre os indivíduos da amostra estudada, uma vez que não apresentavam os critérios diagnósticos para a síndrome completa, que incluem retardo do crescimento pré ou pós-natal, envolvimento do sistema nervoso e dismorfismo facial, além da história de uso de álcool durante a gestação. Dessa forma, é possível supor que o conjunto de características apresentadas por cada indivíduo pode ser considerado como "efeitos alcoólicos fetais"(18) ou associado a outras drogas, ou injúrias.

Uma das características oftalmológicas mais frequentemente encontrada na síndrome alcoólica fetal é a hipoplasia do nervo óptico ${ }^{(10.14-17)}$. Houve, nesse estudo, um caso de hipoplasia do nervo óptico associada com base nasal alargada, epicanto e baixa acuidade visual, mas esses achados também não constituem os critérios para diagnóstico completo da SAF, além do que não há
Tabela 1

\section{Frequência de outras deficiências encontradas nos indivíduos}

\begin{tabular}{lcc}
\hline Deficiências & n & \% \\
\hline Retardo mental grave & 22 & 59,4 \\
Epilepsia & 07 & 18,9 \\
Paralisia cerebral & 07 & 18,9 \\
Dismorfismo facial & 06 & 16,2 \\
Retardo mental leve a moderado & 05 & 13,5 \\
Autismo & 03 & 08,1 \\
Síndrome de Down & 02 & 05,4 \\
Microcefalia & 02 & 05,4 \\
Hidrocefalia & 02 & 05,4 \\
Deficiência auditiva & 02 & 05,4 \\
\hline
\end{tabular}

Tabela 2

Classificação da acuidade visual dos indivíduos conforme o Código Internacional de Doenças - CID-10 da OMS

\begin{tabular}{lcr}
\hline Acuidade visual & n & \% \\
\hline $20 / 20-20 / 60$ & 08 & 33,3 \\
$20 / 70-20 / 200$ & 06 & 25 \\
$<20 / 200$ & 10 & 41,6 \\
\hline
\end{tabular}

Tabela 3

Frequência dos principais achados oftalmológicos dos indivíduos que colaboraram com o exame

\begin{tabular}{lcc}
\hline Achados oftalmológicos & n & $\mathbf{\%}$ \\
\hline Ambliopia & 03 & 12,5 \\
Phthisis bulbi & 03 & 12,5 \\
Erro refrativo & 03 & 12,5 \\
$\quad$ Miopia & 02 & 8,3 \\
$\quad$ Hipermetropia & 01 & 4,1 \\
Astigmatismo & 01 & 4,1 \\
Estrabismo & 02 & 8,3 \\
Ptose & 01 & 4,1 \\
Hipoplasia do nervo óptico & 01 & 4,1 \\
Epicanto & 01 & 4,1 \\
Leucoma & 01 & 4,1 \\
Coroidose miópica & 01 & 4,1 \\
Subluxação do cristalino & 01 & 4,1 \\
\hline
\end{tabular}


relato da genitora do uso de álcool durante a gestação.

A presença das síndromes de Down, Sturge-Weber e Lennox-Gastaut incrementam o estudo com a variedade de diagnósticos encontrados, cada uma apresentando suas próprias manifestações ou associações. A síndrome de Sturge Weber cursa, na maioria das vezes, com glaucoma associado, mas nesse caso não foi possível a realização do exame por ser um indivíduo não colaborativo. Um dos pacientes com síndrome de Down também não colaborou com o exame, sendo portador de retardo mental grave. O outro caso, também com retardo mental grave, apresentava assimetria facial, phthisis bulbi no olho esquerdo e história de hepatite B. O portador da síndrome da rubéola congênita apresentava a principal característica da doença, que é o deficit auditivo bilateral, e também, tinha dismorfismo facial e retardo mental profundo, porém, quanto aos achados oftalmológicos, um olho estava com phthisis bulbi e o outro era microftálmico.

Na maioria dos indivíduos da presente amostra, este foi o primeiro exame oftalmológico realizado. Pode-se verificar uma gama de achados oftalmológicos importantes encontrados nestes indivíduos, o que respalda a sua importância nestes casos. Deve ser ressaltado que em 13 casos o exame mostrou-se muito difícil, havendo deficiências neuropsiquiátricas que impossibilitaram o exame, tendo sido o retardo mental grave diagnosticado na maior parte dos casos. Sabe-se que os indivíduos com necessidades especiais requerem um atendimento especializado e adequado quanto à aparelhagem e recursos humanos ${ }^{(7)}$. Os casos nos quais não foi possível fazer o exame foram encaminhados ao serviço especializado para completar o exame sob narcose.

Elevado percentual de casos apresentava cegueira legal (41,6\%), demonstrando o prejuízo ao desenvolvimento neuropsicomotor enfrentado por esses indivíduos, o que reforça ainda mais a necessidade de um acompanhamento oftalmológico especializado e de equipe especializada. Dados semelhantes foram encontrados anteriormente em outros estudos $(69,5 \% \text { e } 45 \%)^{(9,10)}$. Observam-se divergências entre as pesquisas com portadores de necessidades especiais com relação à acuidade visual. Alguns estudos mostram boa acuidade visual com maior frequência $(44,5 \% \text { e } 61,3 \%)^{(7,11)}$.

O erro refrativo passível de correção mais encontrado foi miopia, presente em dois casos. Há relatos de alta incidência de miopia em portadores de paralisia cerebral por sofrimento fetal ou anóxia ${ }^{(4,5,7),}$ entretanto alguns estudos com crianças com paralisia cerebral e/ou outras necessidades especiais mostram maior frequência de hipermetropia e astigmatismo ${ }^{(6,11-13)}$.

A ambliopia está entre os principais achados oftalmológicos dos indivíduos estudados, sendo também encontrada em outros estudos, que apresentam frequências de ambliopia entre 43 e $74 \%^{(6,7)}$. Nesses estudos, a ambliopia foi encontrada em crianças que apresentavam paralisia cerebral, e apesar da elevada incidência desse diagnóstico na presente pesquisa, os casos de ambliopia não estiveram associados com paralisia cerebral.

A Phthisis bulbi apresentou-se em três casos $(12,5 \%)$, sendo dois bilaterais, o que não foi relatado em outros estudos, podendo justificar um achado ocasional.

A presença de microftalmo em indivíduos com retardo mental ou paralisia cerebral tem sido relatada com frequência na literatura ${ }^{(9,12,14)}$, assim como no presente estudo.

Em indivíduos com necessidades especiais a prevalência de anormalidades oftalmológicas é alta. Entre as mais frequentes estão: erros refrativos, atrofia óptica, nistagmo, estrabismo, retinopatia, ptose e catarata ${ }^{(4-7,11-16)}$.

O leucoma e a subluxação do cristalino foram dois achados oftalmológicos presentes em indivíduo que havia sofrido traumatismo crânioencefálico(TCE).

Todas as anormalidades encontradas nos indivíduos do presente estudo haviam sido previamente descritas, apresentando variação quanto a frequência de estudo a estudo ${ }^{(4,9,12)}$.

É importante salientar que a dificuldade dos profissionais em examinar os indivíduos com severa deficiência mental, pouco colaborativos, de certa forma pode ter mascarado alguns achados oftalmológicos que no momento do exame não foram detectados, e que talvez trouxessem maior riqueza de dados ao estudo em questão. As doenças oftalmológicas dificultam o acesso do paciente às informações do meio ambiente, dificultando a sociabilização, escolaridade e inclusão familiar do paciente.

Os indivíduos incluídos na amostra estudada foram beneficiados, com diagnóstico e tratamento dos casos indicados pelo projeto, com doação de medicamentos, e os casos que requeriam maiores investigações foram encaminhados ao serviço especializado.

\section{Conclusão}

Os achados clínicos múltiplos tais como: doenças neuropsiquiátricas contribuíram para o maior grau de dificuldade na avaliação oftalmológica dos indivíduos. A gama de alterações oftalmológicas detectadas e o grau de comprometimento ocular revelaram a importância do exame oftalmológico em pacientes com necessidades especiais, institucionalizados.

\section{Abstract}

Purpose: To describe the main clinical and visual findings in special needs subjects institutionalized in Pernambuco State. Methods: Thirty seven subjects with special needs, 
institutionalized in Rodolfo Aureliano Community (CRAUR), from Children and Adolescent Foundation (FUNDAC), in Recife PE, were evaluated by a multidiscipline team. The subjects aged 6 to 30 years, with mean of 18.8 5.1 years. Most of them were boys (94.5\%). Institutionalized individuals were considered when they lived in institutions maintained by the State. It was made a crosssectional study, verifying the main clinical and ophthalmic findings of these individuals. It was performed a complete ophthalmic examination in cases with good cooperation in accordance with pre-established protocol. Results: Alcohol use in pregnancy was reported by nine mothers $(24.3 \%)$. Severe mental retardation was seen in 22 subjects $(59.4 \%)$, cerebral palsy and epilepsy in 7 (18.9\%) subjects each. It was possible to perform the complete ophthalmic examination in 24 subjects (64.8\%). Legal blindness was detected in 10 cases $(41.6 \%)$. Main ophthalmologic findings found were amblyopia, refractive error and phthisis bulbi in 3 cases $(12.5 \%)$ each. Strabismus was seen in two cases (8.3\%). Conclusion: The ophthalmological findings detected revealed the importance of ophthalmic examination in institutionalized individuals with special needs. The association with multiple disabilities such as neuropsychiatric diseases contributed to the greatest degree of difficulty in the evaluation and conduct of cases.

Keywords: Fetal alcohol syndrome;Abnormalities, multiple; Disabled persons; Health of institutionalized children; Intitucionalized population; Eye abnormalities

\section{REFERÊNCIAS}

1. Martins CS, Ferriani MG, Silva MA, Zahr NR, Arone KM, Roque EM. Family dynamics from the perspective of parents and children involved in domestic violence against children and adolescents. Rev Latinoam Enferm. 2007;15(5):889-94.

2. Alexandre DT, Vieira ML. Relação de apego entre crianças institucionalizadas que vivem em situação de abrigo. Psicol Estud. 2004;9(2):207-17.

3. Dantas AP, Brandt CT, Leal DNB. Manifestações oculares em pacientes que tiveram desnutrição nos primeiros seis meses de vida. Arq Bras Oftalmol. 2005;68(6):753-6

4. Leal DB, Tavares SS, Ventura LO, Florêncio T. Atendimento a portadores de visão subnormal: estudo retrospectivo de 317 casos. Arq Bras Oftalmol. 1995;58(6):439-42.

5. Mwanza JC, Nikidiaka CM, Kayembe DL, Maillet CY, Mukau EJ, Tuela MR. Ophthalmologic abnormalities in mentally retarded. Bull Soc Belge Ophthalmol. 2000;(277):75-8.

6. Tabuse MKU, Moreira JBC. Estudo das manifestações oculares em crianças com paralisia cerebral. Arq Bras Oftalmol. 1996;59(6):560-6.

7. Celino AC, Trigueiro S, Ventura LO, Toscano J, Barroca R. Alterações oculares em crianças portadoras de paralisia cerebral. Rev Bras Oftalmol. 2003;62(4):248-51.
8. Organização Mundial de Saúde. Classificação estatística internacional de doenças e problemas relacionados à saúde CID-10. São Paulo: Centro Colaborador da Organização Mundial da Saúde para a Classificação de Doenças em Português; 2008 [citado 2008 Dez 16]. Disponível em: http:/ /www.datasus.gov.br/cid10/vol2008/cid10.htm

9. Kocur I, Kuchynka P, Rodný S, Baráková D, Schwartz EC. Causes of severe visual impairment and blindness in children attending schools for the visually handicapped in the Czech Republic. Br J Ophthalmol. 2001;85(10):1149-52. Comment in: Br J Ophthalmol. 2001;85(10):1145-6.

10. Sampaio PR, Carvalho KM, Cagliardo HGRG, Nobre MIR, Botega MBS. Avaliação do retardo do neurodesenvolvimento em crianças especiais em serviço universitário de visão subnormal. Arq Bras Oftalmol. 1999;62(3):235-8.

11. Remígio MC, Leal D, Barros E, Travassos S, Ventura LO. Achados oftalmológicos em pacientes com múltiplas deficiências. Arq Bras Oftalmol. 2006;69(6):929-32.

12. Black PD. Ocular defects in children with cerebral palsy. $\mathrm{Br}$ Med J. 1980;281(6238):487-8.

13. Buckley E, Seaber JH. Dyskinetic strabismus as a sign of cerebral palsy. Am J Ophthalmol. 1981;91(5):652-7.

14. Ordóñez A, Suárez F, Zarante I, Tamayo ML, Contreras G, Jovita $\mathrm{N}$, et al. Un modelo de diagnóstico social y manejo interdisciplinario de una población de niños discapacitados institucionalizados. Univ Med. 2008;49(2):155-71.

15. Strömland K. Ocular abnormalities in the fetal alcohol syndrome. Acta Ophthalmol Suppl. 1985;171:1-50.

16. Cançado Figueiredo M, Carvalho e Silva SR, Preto Guimarães F, Araújo VP. Perfil de pacientes con necesidades especiales. Bol Asoc Argent Odontol Ninõs. 2003;32(1):8-11.

17. Ornoy A, Ergaz Z. Alcohol abuse in pregnant women: effects on the fetus and newborn, mode of action and maternal treatment. Int J Environ Res Public Health. 2010;7(2):364-79. Review.

18. Strömland K. Ocular involvement in the fetal alcohol syndrome. Surv Ophthalmol. 1987;31(4):277-84.

19. Edward DP, Li J, Sawaguchi S, Sugar J, Yue BY, Tso MO. Diffuse corneal clouding in siblings with fetal alcohol syndrome. Am J Ophthalmol. 1993;115(4):484-93.

20. Hellström A, Svensson E, Strömland K. Eye size in healthy Swedish children and in children with fetal alcohol syndrome. Acta Ophthalmol Scand. 1997;75(4):423-8.

21. Pinazo-Duran MD, Renau-Piqueras J, Guerri C, Strömland K. Optic nerve hypoplasia in fetal alcohol syndrome: an update. Eur J Ophthalmol. 1997;7(3):262-70.

22. Strömland K, Pinazo-Durán MD. Optic nerve hypoplasia: comparative effects in children and rats exposed to alcohol during pregnancy. Teratology. 1994;50(2):100-11.
Endereço para correspondência:
Priscilla de Almeida Jorge
Fundação Altino Ventura - FAV
Rua da Soledade, $n^{\circ} 170$ - Boa Vista
CEP: 500070-040 - Recife (PE), Brasil
Fax: (0xx81)3302-4300
E-mail: prialmeida.j@hotmail.com;
fav@fundacaoaltinoventura.org.br 